\title{
TOXICIDAD DEL EXTRACTO ACUOSO, ETANÓLICO Y HEXÁNICO de Annona muricata, Minthostachys mollis, Lupinus mutabilis, Y Chenopodium quinoa SOBRE Tetranychus urticae Y Chrysoperla externa
}

\author{
TOXICITY OF AQUEOUS, ETHANOLIC AND HEXANIC EXTRACTS OF \\ Annona muricata, Minthostachys mollis, Lupinus mutabilis, AND Chenopodium \\ quinoa AGAINST Tetranychus urticae AND Chrysoperla externa
}

\author{
Alfonzo Alegre ${ }^{1}$, José Iannacone ${ }^{1,2 *}$, y Mario Carhuapoma ${ }^{3}$
}

\author{
${ }^{1}$ Universidad Ricardo Palma (URP), Facultad de Ciencias Biológicas (FCB), Laboratorio de Parasitología, \\ Av. Alfredo Benavides 5440, Santiago de Surco, Lima, Perú. \\ ${ }^{2}$ Universidad Nacional Federico Villarreal (UNFV), Facultad de Ciencias Naturales y Matemática \\ (FCCNM), Laboratorio de Ecología y Biodiversidad Animal (LEBA), Jr. Río Chepén 290, El Agustino, \\ Lima, Perú. \\ ${ }^{3}$ Universidad Nacional Mayor de San Marcos (UNMSM), Facultad de Farmacia y Bioquímica (FFB), \\ Distrito de Lima 15001, Lima, Perú. \\ * Autor para correspondencia E-mail: joseiannacone@gmail.com
}

\section{RESUMEN}

La evaluación de la compatibilidad entre los extractos de plantas con propiedades insecticidas y el control biológico de plagas es esencial en estrategias de manejo integrado de plagas. Se evaluó la toxicidad de los extractos acuosos, etanólicos y hexánicos de las hojas de Minthostachys mollis Kunth) Griseb, "muña" y semillas de Annona muricata L. "guanábana", Lupinus mutabilis Sweet, "tarwi" y Chenopodium quinoa Willd "quinua", sobre hembras adultas del ácaro Tetranychus urticae Koch, 1836 "arañita roja", y larvas del primer instar de Chrysoperla externa Hagen, 1861 "león de áfidos". Se emplearon dos concentraciones, 10 y $20 \%$, en un periodo de exposición de 24 y $72 \mathrm{~h}$. Los parámetros de toxicidad se observaron en los valores NOEC (Concentración sin efecto observado) y LOEC (Concentración más baja con efecto observado). El extracto acuoso de M. mollis y el etanólico de $C$. quinoa, ambos al $20 \%$ de concentración, causaron mortalidades en $T$. urticae de 28,9 y $29,6 \%$, respectivamente. El extracto etanólico de $M$. mollis registró la mayor toxicidad de todos los extractos evaluados a las $72 \mathrm{~h}$ de exposición en C. externa, con una mortalidad del 75,7\%. Según el cociente relativo de riesgo de selectividad (CRRS) obtenido a las $48 \mathrm{~h}$ de exposición, los extractos acuosos no representaron un riesgo de toxicidad en C. externa. El extracto acuoso de $M$. mollis y el extracto etanólico de $C$. quinoa, ambos al $20 \%$ de concentración, podrían ser usados en un programa de manejo integrado de T. urticae.

Palabras clave: Arañita roja, bioensayos, plaguicidas botánicos, león de áfidos, toxicidad.

\section{ABSTRACT}

The assessment of the compatibility of plant extracts that have insecticidal properties with pest biological control is essential for the development in integrated pest management strategies. Toxicity of aqueous, ethanolic and hexanic extracts of leaves of Minthostachys mollis (Kunth) Griseb, "muña", and seeds of Annona muricata L. "soursop", Lupinus mutabilis Sweet "tarwi" and Chenopodium quinoa Willd "quinua" were evaluated against adult females of the two-spotted spider mite Tetranychus urticae Koch, 1836, and first instar larvae of green lacewing Chrysoperla externa 
Hagen, 1861. The extracts were applied at concentrations of 10 and $20 \%$ with exposure periods of 24 and $72 \mathrm{~h}$. The parameters of toxicity were observed in the NOEC (no observed effect concentration) and LOEC (lowest observed effect concentration) values. Both the aqueous extract of M. mollis and the ethanolic extract of $C$. quinoa at a concentration of $20 \%$ resulted in mortality rates of 28.9 and $29.6 \%$ of $T$. urticae, respectively. The ethanol extract of $M$. mollis recorded the highest toxicity against C. externa of all extracts evaluated after a $72 \mathrm{~h}$-exposure, with a mortality of $75.7 \%$. According to the RCRS (relative quotient risk of selectivity) obtained at $48 \mathrm{~h}$ of exposure, the aqueous extracts did not show toxicity against $C$. externa. The aqueous $M$. mollis extract and $C$. quinoa ethanolic extract at a concentration of $20 \%$ could be used in an integrated $T$. urticae management program.

Key words: Two spotted mite, bioassays, botanical pesticides, lion aphid, toxicity.

\section{INTRODUCCIÓN}

En la actualidad el uso de agroquímicos es una de las herramientas más empleadas para el control de plagas en la agricultura. Sin embargo, el uso excesivo puede producir contaminación ambiental y desarrollo de plagas resistentes (Campos et al., 2016). Por otra parte, los insecticidas botánicos han sido señalados como una alternativa al uso de insecticidas químicos, ya que suponen un menor riesgo para el ambiente o para la salud humana (Souza et al., 2008). Además, algunos de estos plaguicidas botánicos ya se utilizan como productos de protección vegetal (Campos et al., 2016; Harder et al., 2016).

La infusión de las hojas de Minthostachys mollis (Kunth) Griseb. "Muña" (Lamiaceae), es empleada como un digestivo, antibacteriano, antihelmíntico y afrodisiaco (Hammond et al., 1998; Carhuapoma et al., 2009; TorrenegraAlarcón et al., 2016). Esta planta arbustiva es nativa de Sudamérica, y habita entre 500 y 3500 msnm (Scandaliaris et al., 2007).

Las hojas, semillas y frutos de Annona muricata L. "Guanábana" (Annonaceae), tienen propiedades astringentes, antiespasmódicas, para tratar contusiones y para eliminar piojos (Mayo, 2013). Este árbol frutal es originario de América tropical. El fruto es una baya múltiple o sincarpo, con semillas ovoides comprimidas dorsalmente y de color pardo oscuro brillante (Coto-Alfaro y Saunders, 2001).

Las semillas de Lupinus mutabilis Sweet "Tarwi" (Fabaceae), tienen un alto valor nutricional, y son utilizadas para controlar garrapatas y parásitos intestinales en animales domésticos, debido a la presencia de alcaloides. Esta planta es una leguminosa que se cultiva habitualmente en las zonas altoandinas de Sudamérica (Jacobsen y Mujica, 2006).

Los granos de Chenopodium quinoa Willd "Quinua" (Amaranthaceae), son usados en la alimentación humana debido a sus proteínas, aminoácidos esenciales y ácidos grasos. Además se le atribuye propiedades cicatrizantes, desinflamantes, analgésicas y desinfectantes
(Mujica y Jacobsen, 2006). Esta planta anual es cultivada en mayor cantidad en los Andes de Perú y Bolivia.

Entre los insectos, Chrysoperla externa Hagen 1861, "león de áfidos", es un depredador eficaz en el manejo ecológico e integrado de plagas, pudiendo habitar un gran número de agroecosistemas (Núñez, 1988; Aud et al., 2001; Iannacone et al., 2007). Las larvas son muy voraces y se alimentan preferentemente de insectos de cuerpo blando (Soto y Iannacone, 2008). Por otro lado, se ha demostrado su sensibilidad frente a diversos pesticidas (Silva et al., 2012; Moura et al., 2012), así como a productos botánicos (Iannacone y Lamas, 2002; Medina et al., 2004; Iannacone et al., 2015).

Por otra parte, Tetranychus urticae Koch, 1836, "arañita roja", es un ácaro polífago y cosmopolita que perjudica diversos cultivos de importancia económica a nivel mundial (Jeppson et al., 1975, de Moraes y Flechtmann, 2008). Este ácaro presenta una rápida resistencia al uso de pesticidas, debido a que estos individuos tienen un ciclo de vida corto, abundante descendencia y reproducción de tipo arrenótoca (Van Leeuwen et al., 2010).

La evaluación de la compatibilidad entre los extractos de plantas con propiedades insecticidas y el control biológico de plagas son esenciales para una estrategia de manejo integrado de $T$. urticae (Iannacone et al., 2015; Parolin et al., 2015).

El objetivo principal de este estudio fue evaluar la toxicidad del extracto acuoso, etanólico y hexánico de las hojas de Minthostachys mollis "muña", semillas de Annona muricata "guanábana", Lupinus mutabilis "tarwi", y Chenopodium quinoa "quinua" sobre Tetranychus urticae y Chrysoperla externa para un posible uso en Manejo Integrado de Plagas (MIP).

\section{MATERIALES Y MÉTODOS}

Los bioensayos toxicológicos se realizaron en el Laboratorio de Parasitología de la Universidad Ricardo Palma (URP), Distrito de Santiago de Surco, Lima, Perú, durante el 2016. 


\section{Obtención del material vegetal}

El material vegetal se adquirió en el mercado mayorista de Santa Anita, Lima, Perú. Se compraron $2 \mathrm{~kg}$ de plantas enteras de M. mollis provenientes de la ciudad de Concepción (Junín), ubicada a $3286 \mathrm{msnm}$. Las semillas de A. muricata se separaron de $3 \mathrm{~kg}$ de fruta madura. Finalmente, se consiguieron $500 \mathrm{~g}$ de semillas no lavadas de $C$. quinoa var. Blanca de Junín y $500 \mathrm{~g}$ de semillas no lavadas de L. mutabilis, provenientes de la ciudad de Sapallanga (Junín), ubicada a $3299 \mathrm{msnm}$. El material vegetal fue certificado por el Laboratorio de Diversidad-Vegetal de la Facultad de Ciencias Naturales y Matemática de la Universidad Nacional Federico Villarreal (UNFV), según el Sistema de Clasificación Cronquist (1981).

\section{Obtención del material animal}

Los huevecillos maduros de C. externa (3 millares) se adquirieron del Programa Nacional de Control Biológico, Servicio Nacional de Sanidad Agraria (PN-CB-SENASA), Lima, Perú, y se acondicionaron en tres recipientes plásticos de $8 \mathrm{~L}$ de capacidad hasta el momento de la eclosión. Las larvas del primer instar $(<24$ h) recién emergidas se alimentaron con huevos de Sitotroga cerealella (Olivier, 1789) antes de su utilización en los bioensayos (Iannacone y Alvariño, 2010).

Los especímenes de $T$. urticae se recolectaron semanalmente de plantas de Prunus persica L. "durazno", no tratadas previamente con insecticidas o acaricidas sintéticos, de parques del distrito de Santa Anita. Se usaron para los bioensayos únicamente hembras adultas de $T$. urticae, debido a que es uno de los estados de desarrollo más susceptible. La identificación de la especie se realizó en el Laboratorio de Entomología del Centro de Diagnóstico de Sanidad Vegetal del Servicio Nacional de Sanidad Agraria (SENASA), Lima, Perú. Se usaron solo ácaros machos para la confirmación de la especie (Ferragut y Santonja, 1989).

\section{Extractos vegetales}

Las semillas de A. muricata y hojas de M. mollis se lavaron con agua potable para la remoción de las impurezas presentes, luego se secaron y colocaron en una estufa a $40^{\circ} \mathrm{C}$ por $48 \mathrm{~h}$. Posteriormente se procedió a retirar el material vegetal de la estufa. Luego se removió la testa de las semillas de $A$. muricata, para obtener solo el endospermo. A continuación se procedió a triturar por separado el material vegetal seco con un mortero hasta la obtención de un polvillo fino. El tamaño de las partículas granulométricas del polvillo de las dos especies botánicas se determinó por un tamizaje de $250 \mathrm{u}$ (US mesh =60). Las semillas de C. quinoa y L. mutabilis no se sometieron al procedimiento descrito anteriormente de triturado y obtención de un polvillo fino, sino que previa limpieza de las impurezas presentes, las semillas se secaron en estufa. Finalmente, el material vegetal se colectó por separado en frascos de vidrio ámbar hasta la preparación de los extractos.

El extracto acuoso se obtuvo luego de pesar 20 g del material vegetal de $M$. mollis, A. muricata, $C$. quinoa y L. mutabilis con $100 \mathrm{~mL}$ de agua purificada no gasificada (Cielo ${ }$, AJEPER, Santiago de Surco, Lima, Perú) $(\mathrm{pH}=7,79$, potencial redox $=$ $55 \mathrm{mV}$, conductividad $=54,5 \mathrm{uScm}^{-2}$, temperatura $=25^{\circ} \mathrm{C}, \%$ saturación de oxigeno $=76,3 \%$, Oxígeno disuelto $\left.=6,17 \mathrm{mg} \mathrm{L}^{-1}\right)$ por $24 \mathrm{~h}$ a temperatura ambiente (Iannacone y Quispe, 2004). El extracto etanólico se obtuvo luego de pesar $20 \mathrm{~g}$ del material vegetal de $M$. mollis, $A$. muricata, C. quinoa y L. mutabilis con $100 \mathrm{~mL}$ de etanol al $70 \%$ por 72 $\mathrm{h}$ a temperatura ambiente. El extracto hexánico se obtuvo luego de pesar $20 \mathrm{~g}$ del material vegetal de M. mollis y A. muricata con $100 \mathrm{~mL}$ de n-hexano a las mismas condiciones señaladas con el extracto etanólico. No se trataron las semillas de C. quinoa y L. mutabilis con este solvente apolar.

Los extractos botánicos se agitaron manualmente cada $8 \mathrm{~h}$ por 3 días durante el proceso de extracción. Transcurrido este tiempo, se filtraron usando un papel filtro (Whatman $\mathrm{N}^{\circ} 1$ ). A partir del extracto inicial del $20 \%$ de concentración, se hizo una dilución para obtener la concentración del 10\%. Finalmente, cada extracto vegetal se almacenó en frascos de color ámbar para su utilización en los bioensayos.

\section{Bioensayos}

Los bioensayos se realizaron por contacto residual en envases plásticos de $12 \mathrm{~mL}$ de capacidad (Iannacone et al., 2015), a los cuales se les hizo pequeños orificios en la tapa para permitir la respiración de ambos artrópodos. El protocolo consistió en pasar un hisopo estéril previamente sumergido por 30 seg una sola vez en cada extracto vegetal, para luego ser esparcido homogéneamente por todas las paredes de cada recipiente plástico. Luego los envases con solvente etanólico y hexánico se secaron a temperatura ambiente por un intervalo de 3-4 h, mientras que los envases con solvente acuoso fue por $2 \mathrm{~h}$ (Iannacone y Alvariño, 2010).

Se utilizaron 10 hembras adultas de T. urticae por cada recipiente plástico y no se le suministró alimento durante la realización del bioensayo. Diez larvas del primer instar $(<24 \mathrm{~h})$ de $C$. externa se colocaron individualmente en cada recipiente plástico, debido a que estos insectos presentan hábitos de canibalismo cuando escasea el alimento (Núñez, 1988).

La temperatura y humedad relativa promedio 
del laboratorio fue de $28,6 \pm 1{ }^{\circ} \mathrm{C}$ y $67 \pm 2 \%$, respectivamente. Los bioensayos se realizaron en condiciones de oscuridad para evitar la fotólisis de los extractos botánicos (Iannacone y Lamas, 2003b; Iannacone y Alvariño, 2005). Las lecturas de mortalidad fueron a las $24 \mathrm{y} 48 \mathrm{~h}$ para T. urticae, y a las 24,48 y $72 \mathrm{~h}$ para C. externa. No se completó el bioensayo hasta las $72 \mathrm{~h}$ con T. urticae, debido a que hubo una mortalidad superior al $20 \%$ en el control. Se evidenció la muerte de ambas especies cuando estas no realizaron algún movimiento corporal coordinado al ser estimulados con un alfiler entomológico durante $15 \mathrm{~s}$ en su observación en el microscopio estereoscópico (Iannacone y Alvariño, 2005).

\section{Diseño experimental y análisis de datos}

Se usaron dos concentraciones (20\% y $10 \%)$ de los extractos botánicos acuosos, etanólicos y hexánicos para las pruebas de toxicidad con T. urticae y C. externa, cada uno con cuatro repeticiones y un control de agua purificada no gasificada, alcohol etílico al 70\% y n-hexano. El control no debió superar el $20 \%$ de mortalidad en los bioensayos. Se hizo un ajuste del control a $0 \%$ mediante la fórmula de Schneider-Orelli's derivada de la fórmula de Abbott (Fusé et al., 2013), luego los datos obtenidos se transformaron según la expresión $\sqrt{x+1}$. Los estadísticos descriptivos se calcularon con el programa estadístico IBM SPSS Statistics versión 23. Se realizó el análisis de varianza (ANDEVA) y la prueba post hoc de Duncan con un nivel de significancia de $\mathrm{p} \leq$ 0,05 , además se calcularon los valores de LOEC (Concentración más baja con efecto observado) y NOEC (Concentración sin efecto observado). Para todos los extractos a las $48 \mathrm{~h}$ de exposición, al ser la mortalidad de las hembras adultas de T. urticae y de las larvas del primer instar de $C$. externa, en la concentración del 10\% del extracto estadísticamente igual al control, no necesitó adicionar una concentración al 5\%, que es lo sugerido para calcular los valores de NOEC y LOEC (Castillo, 2004). Finalmente, se determinó el cociente relativo de riesgo de selectividad (CRRS) adaptado de Bahlai et al. (2010), y Stanley y Preetha (2016) empleando la relación presa (T. urticae)/ depredador (C. externa) al no tenerse la dosis de aplicación recomendada en campo de los cuatro extractos botánicos para el control de T. urticae en diferentes cultivos agrícolas. El CRRS se calculó luego de dividir el porcentaje de mortalidad de T. urticae/C. externa a las $48 \mathrm{~h}$ de exposición en los bioensayos con ambas especies. Los valores mayores a 1 no representaron un riesgo adverso en la mortalidad de C. externa, mientras que los valores menores a 1 si ocasionaron un riesgo negativo en el depredador.

\section{Análisis fitoquímico}

Se realizó utilizando procedimientos estandarizados para la detección de alcaloides (Dragendorff), flavonoides (Shinoda), esteroides (Liebermann Burchard), grupos fenólicos (Tricloruro férrico) y saponinas (producción de espuma) (Lock, 1994). La presencia de metabolitos secundarios se detectó en tres categorías: Muy abundante $(++++)$, Abundante $(+++)$, moderada $(++)$, leve $(+)$, ausencia $(-)$, dudoso $( \pm)$.

\section{RESULTADOS Y DISCUSIÓN}

El extracto acuoso de M. mollis no presentó efectos acaricidas en hembras adultas de T. urticae a las $24 \mathrm{~h}$ de exposición y al $20 \%$ de concentración. Los restantes extractos acuosos de las plantas evaluadas en los bioensayos presentaron efecto acaricida (Tabla 1). A las $48 \mathrm{~h}$ de exposición se observó un incremento de la mortalidad de ácaros, siendo el extracto acuoso de $M$. mollis al $20 \%$ de concentración el que presentó la mayor actividad acaricida (29,3\%). La Tabla 2 muestra la presencia de los principales metabolitos secundarios evidenciados mediante el análisis fitoquímico. Se detectó la presencia de alcaloides, flavonoides, esteroides, grupos fenólicos y saponinas, en los extractos botánicos empleados en los bioensayos (Dadé et al., 2009). Iannacone y Lamas (2003a) indicaron la actividad repelente e insecticida de Minthostachys sp por infusión, planta y polvo seco en el lepidóptero Phthorimaea operculella (Zeller, 1873), además L. mutabilis presentó solo actividad insecticida por infusión y macerado.

Los extractos etanólicos de A. muricata y $M$. mollis en ambas concentraciones y a las $24 \mathrm{~h}$ de exposición no presentaron actividad acaricida, en cambio, los extractos de L. mutabilis y C. quinoa si presentaron efecto acaricida de 3,6\% y $6,1 \%$, al $20 \%$ de concentración, respectivamente (Tabla 3 ). A las 48 h de exposición se observó que el extracto de C. quinoa mostró la mayor actividad acaricida, la cual disminuyó a las $48 \mathrm{~h}$ de exposición por aumento de la mortalidad del testigo antes previo al ajuste del control con la fórmula SchneiderOrelli's (Tabla 3). Los metabolitos secundarios de esta especie botánica presentan actividad biológica debido principalmente a la presencia de saponinas (Woldemichael y Wink, 2001, Guzmán et al., 2015). Los granos de quinua pueden tener una concentración de 0 a $6 \%$ de saponinas, según la variedad (Iannacone y Quispe, 2004). Tava y Odoardi (1996) comprobaron la actividad insecticida de las saponinas de Medicago spp. sobre larvas del lepidóptero Lobesia botrana (Denis y Schiffermüller, 1775). Harder et al. (2016) evaluaron el efecto acaricida del extracto etanólico crudo de semillas de $C$. quinoa en 
Tabla 1. Toxicidad del extracto acuoso de Annona muricata, Minthostachys mollis, Lupinus mutabilis, y Chenopodium quinoa sobre Tetranychus urticae (Trombidiformes: Tetranychidae).

Table 1. Toxicity of the aqueous extract of Annona muricata, Minthostachys mollis, Lupinus mutabilis, and Chenopodium quinoa against Tetranychus urticae (Trombidiformes: Tetranychidae).

\begin{tabular}{|c|c|c|c|c|c|c|c|c|}
\hline \multirow[b]{3}{*}{ Concentración } & \multirow{2}{*}{\multicolumn{2}{|c|}{ A. muricata }} & \multicolumn{2}{|c|}{ M. mollis } & \multicolumn{2}{|c|}{ L. mutabilis } & \multicolumn{2}{|c|}{ C. quinoa } \\
\hline & & & & Exp & ción & & & \\
\hline & $24 \mathrm{~h}$ & $48 \mathrm{~h}$ & $24 \mathrm{~h}$ & $48 \mathrm{~h}$ & $24 \mathrm{~h}$ & $48 \mathrm{~h}$ & $24 \mathrm{~h}$ & $48 \mathrm{~h}$ \\
\hline Control & $0 \mathrm{a}$ & $0 \mathrm{a}$ & $0 \mathrm{a}$ & $0 \mathrm{a}$ & $0 \mathrm{a}$ & $0 \mathrm{a}$ & $0 \mathrm{a}$ & $0 \mathrm{a}$ \\
\hline $10 \%$ & $7,6 a b$ & $16,6 b$ & $2,5 \mathrm{a}$ & $5,5 a$ & $5,1 \mathrm{ab}$ & $8,3 a$ & $17,9 b$ & $13,8 b$ \\
\hline $20 \%$ & $17,9 b$ & $22,2 b$ & $7,6 a$ & $29,6 b$ & $17,9 b$ & $22,2 b$ & $10,2 b$ & $16,6 b$ \\
\hline NOEC (\%) & 10 & $<10$ & 20 & 10 & 10 & 10 & $<10$ & $<10$ \\
\hline LOEC (\%) & 20 & 10 & $>20$ & 20 & 20 & 20 & 10 & 10 \\
\hline $\mathrm{F}$ & 5,7 & 10,9 & 2,2 & 13,9 & 5,05 & 12,0 & 10,4 & 5,2 \\
\hline Sig. & 0,02 & 0,01 & 0,17 & 0,00 & 0,03 & 0,00 & 0,00 & 0,03 \\
\hline
\end{tabular}

Letras minúsculas iguales en una misma columna representan que no hay diferencia significativa entre los valores por la prueba de Duncan $(\mathrm{p} \leq 0,05)$.

NOEC $=$ Concentración sin efecto observado. LOEC $=$ Concentración mínima con efecto observado. $\mathrm{F}=$ Prueba de Fisher. Sig. = Nivel de significancia.

Tabla 2. Identificación de los principales metabolitos secundarios evidenciados en los extractos botánicos.

Table 2. Identification of the main secondary metabolites found in the botanical extracts.

\begin{tabular}{lccccc}
\hline Extracto/prueba & Dragendorff & Shinoda & $\begin{array}{c}\text { Liebermann } \\
\text {-Burchard }\end{array}$ & $\begin{array}{c}\text { Tricloruro } \\
\text { férrico }\end{array}$ & $\begin{array}{c}\text { Producción } \\
\text { de espuma }\end{array}$ \\
\hline M. mollis acuoso & - & +++ & - & ++++ & ++ \\
M. mollis etanólico & - & ++ & - & ++++ & ++ \\
M. mollis hexánico & - & - & ++ & + & - \\
\hline A. muricata acuoso & - & - & - & - & + \\
A. muricata etanólico & + & - & - & - & + \\
A. muricata hexánico & ++ & - & + & - & + \\
\hline C. quinoa acuoso & - & - & - & ++ & ++ \\
C. quinoa etanólico & + & + & - & ++ & + \\
\hline L. mutabilis acuoso & +++ & + & - & Grupos & Saponinas \\
L. mutabilis etanólico & + & ++ & flavonoides & Esteroides & frólicos \\
\hline Grupo funcional & Alcaloides & Flapos & + \\
\hline
\end{tabular}

$++++=$ Muy abundante,$+++=$ abundante,$++=$ moderada,$+=$ escasa, $-=$ ausencia,$\pm=$ dudoso.

hembras adultas de T. urticae por contacto residual, produciendo mortalidades entre el 89$91 \%$ a las $72 \mathrm{~h}$ de exposición con concentraciones de 7,6 y $9,1 \%(p / v)$. Se puede diferir con los autores según los métodos empleados, ya que ellos obtuvieron el extracto crudo de semillas a través de una extracción en Soxhlet, mientras que en este trabajo se usó la maceración de semillas en etanol, produciendo una mortalidad del $29 \%$ de los ácaros a las $24 \mathrm{~h}$ de exposición. Además, cabe mencionar que según el reporte de Gandarillas (1967), las semillas de C. quinoa var. Blanca de
Junín presentan un bajo contenido de saponinas, lo que podría ser un factor determinante para sustentar la baja actividad acaricida menor al 20\% según el criterio señalado por Stanley y Preetha (2016). Por otro lado, Iannacone y Quispe (2004) comprobaron una actividad insecticida de 7,5\% de los extractos acuosos de C. quinoa sobre adultos del coleóptero Sitophilus zeamais (Motschulsky, 1855) en ensayos de contacto en papel filtro y con alimento. Russo et al. (2011) evaluaron el extracto acuoso de Chenopodium album L. en adultos y larvas del coleóptero Oryzaephilus surinamensis 
Tabla 3. Toxicidad del extracto etanólico de Annona muricata, Minthostachys mollis, Lupinus mutabilis, y Chenopodium quinoa sobre Tetranychus urticae (Trombidiformes: Tetranychidae).

Table 3. Toxicity of the ethanolic extract of Annona muricata, Minthostachys mollis, Lupinus mutabilis, and Chenopodium quinoa against Tetranychus urticae (Trombidiformes: Tetranychidae).

A. muricata
M. mollis

Exposición

\begin{tabular}{|c|c|c|c|c|c|c|c|c|}
\hline Concentración & $24 \mathrm{~h}$ & $48 \mathrm{~h}$ & $24 \mathrm{~h}$ & $48 \mathrm{~h}$ & $24 \mathrm{~h}$ & $48 \mathrm{~h}$ & $24 \mathrm{~h}$ & $48 \mathrm{~h}$ \\
\hline Control & $0 \mathrm{a}$ & $0 \mathrm{a}$ & $0 \mathrm{a}$ & $0 \mathrm{a}$ & $0 \mathrm{a}$ & $0 \mathrm{a}$ & $0 \mathrm{a}$ & $0 \mathrm{a}$ \\
\hline $10 \%$ & $6,1 \mathrm{a}$ & $8,3 a$ & $1,0 \mathrm{a}$ & $8,3 a b$ & $6,1 \mathrm{ab}$ & $11,1 b$ & $3,6 a$ & $11,1 b$ \\
\hline $20 \%$ & $3,6 a$ & $11,1 \mathrm{a}$ & $6,1 \mathrm{a}$ & $13,8 b$ & $8,6 b$ & $13,8 b$ & $28,9 b$ & $18,5 b$ \\
\hline NOEC (\%) & 20 & 20 & 20 & 10 & 10 & $<10$ & 10 & $<10$ \\
\hline LOEC (\%) & $>20$ & $>20$ & $>20$ & 20 & 20 & 10 & 20 & 10 \\
\hline $\mathrm{F}$ & 2,2 & 2,2 & 2,4 & 6,0 & 3,6 & 4,5 & 17,6 & 10,5 \\
\hline Sig. & 0,1 & 0,1 & 0,1 & 0,01 & 0,05 & 0,03 & 0,00 & 0,00 \\
\hline
\end{tabular}

Letras minúsculas iguales en una misma columna representan que no hay diferencia significativa entre los valores por la prueba de Duncan $(\mathrm{p} \leq 0,05)$.

NOEC $=$ Concentración sin efecto observado. LOEC $=$ Concentración mínima con efecto observado. $\mathrm{F}=$ Prueba de Fisher. Sig. = Nivel de significancia.

(Linnaeus, 1758) por medio de ingestión y obtuvieron un menor porcentaje de supervivencia en ambos estados de desarrollo. Los resultados obtenidos por Zegarra (2010) evidenciaron la actividad biológica del aceite esencial de $C$. quinoa var. Markjo en la oviposición de hembras y eclosión del ácaro Boophilus microplus (Canestrini, 1888).

Los extractos hexánicos de A. muricata y $M$. mollis no presentaron efecto acaricida significativo en comparación al control a las 24 y $48 \mathrm{~h}$ de exposición, en ambas concentraciones evaluadas (Tabla 4). Sin embargo, los resultados del presente trabajo difieren de los obtenidos por Maciel et al. (2015), quienes encontraron una mayor actividad acaricida del extracto hexánico de semillas de $A$. muricata en hembras adultas de T. urticae. Esto podría deberse al proceso de extracción, a las concentraciones empleadas y al método del bioensayo de toxicidad. Por otro lado, hasta la fecha no se tiene información sobre el efecto del extracto hexánico de M. mollis en este ácaro.

Los extractos acuosos de las cuatro especies botánicas evaluadas no presentaron efecto insecticida en larvas del primer instar de C. externa a las 24 h de exposición. A las 48 h de exposición se observó un efecto insecticida de 14,2\% y 17,8\% de los extractos de A. muricata y L. mutabilis. A las 72 h de exposición la mortalidad se elevó a 30,3\% y $63,64 \%$ para los cuatro extractos botánicos, siendo el extracto de M. mollis al 20\% de concentración el que produjo la mayor actividad insecticida, mientras que el extracto de C. quinoa al $10 \%$ de concentración registró la menor actividad insecticida con este solvente (Tabla 5). La toxicidad del extracto acuoso crudo de semillas de Annona squamosa L. se reportó anteriormente por Leatemia e Isman (2004), quienes indicaron actividad insecticida sobre larvas del primer instar de Chrysoperla carnea (Stephens, 1836) a las 24 y 48 h de exposición por contacto residual.

Los extractos etanólicos no presentaron efecto insecticida sobre C. externa a las $24 \mathrm{~h}$ de exposición. A las 48 h de exposición se observó un efecto insecticida de $15,2 \%$ con el extracto de C. quinoa (Tabla 6). A las $72 \mathrm{~h}$ de exposición la mortalidad sobre C. externa se elevó entre $43,3 \%$ y $75,6 \%$ para los cuatro extractos botánicos, siendo el extracto de $M$. mollis al $20 \%$ de concentración el que presentó la mayor actividad insecticida registrada en los bioensayos, mientras que el extracto de L. mutabilis al $10 \%$ de concentración presentó la menor actividad insecticida (Tabla 6). El análisis fitoquímico del extracto etanólico de M. mollis evidenció la presencia de flavonoides, grupos fenólicos y saponinas como los posibles metabolitos secundarios con actividad insecticida (Tabla 2). No se cuenta en la actualidad con información acerca de la actividad insecticida de esta especie botánica en C. externa.

Los extractos hexánicos de A. muricata y $M$. mollis no presentaron efecto insecticida significativo en $C$. externa en comparación con el control a las 24 y $48 \mathrm{~h}$ de evaluación. A las $72 \mathrm{~h}$ de exposición, solo el extracto de M. mollis produjo mortalidad de $23,5 \%$ y $26,4 \%$, con ambas concentraciones evaluadas (Tabla 7). Se podría 
Tabla 4. Toxicidad del extracto hexánico de Annona muricata, y Minthostachys mollis, sobre Tetranychus urticae (Trombidiformes: Tetranychidae).

Table 4. Toxicity of the hexanic extract of Annona muricata, and Minthostachys mollis, against Tetranychus urticae (Trombidiformes: Tetranychidae).

\begin{tabular}{lccccc}
\hline & \multicolumn{2}{c}{ A. muricata } & & \multicolumn{2}{c}{ M. mollis } \\
Concentración & $\mathbf{2 4} \mathbf{h}$ & $\mathbf{4 8} \mathbf{h}$ & $\mathbf{2 4} \mathbf{h}$ & $\mathbf{4 8 ~} \mathbf{~}$ \\
\hline Control & $0 \mathrm{a}$ & $0 \mathrm{a}$ & & $0 \mathrm{a}$ & $0 \mathrm{a}$ \\
$10 \%$ & $0 \mathrm{a}$ & $0 \mathrm{a}$ & & $0 \mathrm{a}$ & $0 \mathrm{a}$ \\
$20 \%$ & $0 \mathrm{a}$ & $0 \mathrm{a}$ & & $2,4 \mathrm{a}$ & $6,6 \mathrm{a}$ \\
NOEC $(\%)$ & 20 & 20 & & 20 & 20 \\
LOEC $(\%)$ & $>20$ & $>20$ & & $>20$ & $>20$ \\
F & 0,2 & 1,4 & & 3,0 & 3,8 \\
Sig. & 0,7 & 0,2 & & 0,1 & 0,06
\end{tabular}

Letras minúsculas iguales en una misma columna representan que no hay diferencia significativa entre los valores por la prueba de Duncan $(\mathrm{p} \leq 0,05)$.

NOEC $=$ Concentración sin efecto observado. LOEC $=$ Concentración mínima con efecto observado. $\mathrm{F}=$ Prueba de Fisher. Sig. = Nivel de significancia.

Tabla 5. Toxicidad del extracto acuoso de Annona muricata, Minthostachys mollis, Lupinus mutabilis, y Chenopodium quinoa sobre Chrysoperla externa (Neuroptera: Chrysopidae).

Table 5. Toxicity of the aqueous extract of Annona muricata, Minthostachys mollis, Lupinus mutabilis, and Chenopodium quinoa against Chrysoperla externa (Neuroptera: Chrysopidae).

\begin{tabular}{|c|c|c|c|c|c|c|c|c|c|c|c|c|}
\hline \multirow[b]{3}{*}{ Concentración } & \multirow{2}{*}{\multicolumn{3}{|c|}{ A. muricata }} & \multirow{2}{*}{\multicolumn{3}{|c|}{ M. mollis }} & \multicolumn{3}{|c|}{ L. mutabilis } & \multicolumn{3}{|c|}{ C. quinoa } \\
\hline & & & & & & & sición & & & & & \\
\hline & $24 \mathrm{~h}$ & $48 \mathrm{~h}$ & $72 \mathrm{~h}$ & $24 \mathrm{~h}$ & $48 \mathrm{~h}$ & $72 \mathrm{~h}$ & $24 \mathrm{~h}$ & $48 \mathrm{~h}$ & $72 \mathrm{~h}$ & $24 \mathrm{~h}$ & $48 \mathrm{~h}$ & $72 \mathrm{~h}$ \\
\hline Control & $0 \mathrm{a}$ & $0 \mathrm{a}$ & $0 \mathrm{a}$ & $0 \mathrm{a}$ & $0 \mathrm{a}$ & $0 \mathrm{a}$ & $0 \mathrm{a}$ & $0 \mathrm{a}$ & $0 \mathrm{a}$ & $0 \mathrm{a}$ & $0 \mathrm{a}$ & $0 \mathrm{a}$ \\
\hline $10 \%$ & $2,5 \mathrm{a}$ & $11,6 \mathrm{~b}$ & $36,3 b$ & $7,6 a$ & $8,9 a$ & $59,6 \mathrm{~b}$ & $0 \mathrm{a}$ & $0 \mathrm{a}$ & $31,3 \mathrm{~b}$ & $0 \mathrm{a}$ & $0,8 \mathrm{a}$ & $24,2 b$ \\
\hline $20 \%$ & $7,6 a$ & $14,2 b$ & $54,5 b$ & $0 \mathrm{a}$ & $14,2 \mathrm{a}$ & $63,6 b$ & $5,1 \mathrm{a}$ & $17,8 b$ & $47,4 \mathrm{~b}$ & $5,1 \mathrm{a}$ & $6.2 \mathrm{a}$ & $30,3 \mathrm{~b}$ \\
\hline NOEC (\%) & 20 & $<10$ & $<10$ & 20 & 20 & $<10$ & 20 & 10 & $<10$ & 20 & 20 & $<10$ \\
\hline LOEC (\%) & $>20$ & 10 & 10 & $>20$ & $>20$ & 10 & $>20$ & 20 & 10 & $>20$ & $>20$ & 10 \\
\hline $\mathrm{F}$ & 0,9 & 4,8 & 6,8 & 2,2 & 2,7 & 28,4 & 0,7 & 4,3 & 11,7 & 3,4 & 1,2 & 5,2 \\
\hline Sig. & 0,4 & 0,03 & 0,02 & 0,1 & 0,1 & 0,00 & 0,5 & 0,04 & 0,01 & 0,06 & 0,3 & 0,03 \\
\hline
\end{tabular}

Letras minúsculas iguales en una misma columna representan que no hay diferencia significativa entre los valores por la prueba de Duncan $(\mathrm{p} \leq 0,05)$.

NOEC $=$ Concentración sin efecto observado. LOEC $=$ Concentración mínima con efecto observado. $\mathrm{F}=$ Prueba de Fisher. Sig. = Nivel de significancia.

indicar que los metabolitos secundarios extraídos con este solvente apolar con estas concentraciones no son perjudiciales para este insecto como se ha observado con otras especies botánicas (Iannacone et al. 2007)

Los cocientes relativos de riesgo de selectividad (CRRSs) obtenidos con los extractos acuosos y etanólicos indican que éstos no produjeron un riesgo en la mortalidad de las larvas del primer instar de C. externa. Sin embargo, el CRRS del extracto hexánico de M. mollis al $20 \%$ de concentración evidenció un riesgo en la mortalidad de $C$. externa (Tabla 8).
El menor valor del CRRS se observó en el extracto acuoso de M. mollis al $20 \%$ de concentración y a las 48 h de evaluación (Tabla 9). Este resultado indicó que a esta concentración, el extracto botánico no ocasionó un riesgo en la mortalidad de larvas del primer instar de C. externa en los bioensayos de toxicidad, además cabe mencionar que a esta concentración se observó un efecto acaricida del 30\% de mortalidad en hembras adultas de $T$. urticae. Varios trabajos en la literatura científica han evaluado la selectividad de los extractos de plantas con propiedades insecticidas en los agentes de control biológico para evaluar su compati- 
Tabla 6. Toxicidad del extracto etanólico de Annona muricata, Minthostachys mollis, Lupinus mutabilis, y Chenopodium quinoa sobre Chrysoperla externa (Neuroptera: Chrysopidae).

Table 6. Toxicity of the ethanolic extract of Annona muricata, Minthostachys mollis, Lupinus mutabilis, and Chenopodium quinoa against Chrysoperla externa (Neuroptera: Chrysopidae).

\begin{tabular}{|c|c|c|c|c|c|c|c|c|c|c|c|c|}
\hline \multirow[b]{3}{*}{ Concentración } & \multirow{2}{*}{\multicolumn{3}{|c|}{ A. muricata }} & \multirow{2}{*}{\multicolumn{2}{|c|}{ M. mollis }} & \multicolumn{4}{|c|}{ L. mutabilis } & \multicolumn{3}{|c|}{ C. quinoa } \\
\hline & & & & & & Expo & ición & & & & & \\
\hline & $24 \mathrm{~h}$ & $48 \mathrm{~h}$ & $72 \mathrm{~h}$ & $24 \mathrm{~h}$ & $48 \mathrm{~h}$ & $72 \mathrm{~h}$ & $24 \mathrm{~h}$ & $48 \mathrm{~h}$ & $72 \mathrm{~h}$ & $24 \mathrm{~h}$ & $48 \mathrm{~h}$ & $72 \mathrm{~h}$ \\
\hline Control & $0 \mathrm{a}$ & 0a & $0 \mathrm{a}$ & 0a & $0 a$ & $0 \mathrm{a}$ & $0 \mathrm{a}$ & $0 \mathrm{a}$ & $0 \mathrm{a}$ & $0 a$ & $0 \mathrm{a}$ & $0 \mathrm{a}$ \\
\hline $10 \%$ & $0 \mathrm{a}$ & $7,0 \mathrm{a}$ & $54,5 \mathrm{~b}$ & $0 \mathrm{a}$ & $0 \mathrm{a}$ & $42,4 b$ & $0 \mathrm{a}$ & $4,3 a$ & $39,3 b$ & $0 \mathrm{a}$ & $0 \mathrm{a}$ & $48,4 \mathrm{~b}$ \\
\hline $20 \%$ & $5,2 \mathrm{a}$ & $12,5 a$ & $60,6 b$ & $0 \mathrm{a}$ & $7,0 \mathrm{a}$ & $75,7 \mathrm{c}$ & $0 \mathrm{a}$ & $9,7 a$ & $43,3 b$ & $0 \mathrm{a}$ & $15,2 b$ & $51,5 b$ \\
\hline NOEC (\%) & 20 & 20 & $<10$ & 20 & 20 & $<10$ & 20 & 20 & $<10$ & 20 & 10 & $<10$ \\
\hline LOEC (\%) & $>20$ & $>20$ & 10 & $>20$ & $>20$ & 10 & $>20$ & $>20$ & 10 & $>20$ & 20 & 10 \\
\hline $\mathrm{F}$ & 0,6 & 3,1 & 36,7 & 1,1 & 1,3 & 65,2 & 0,3 & 1,2 & 25,6 & 1,6 & 4,6 & 28,2 \\
\hline Sig. & 0,5 & 0,08 & 0,00 & 0,3 & 0,3 & 0,00 & 0,7 & 0,3 & 0,00 & 0,2 & 0,03 & 0,00 \\
\hline
\end{tabular}

Letras minúsculas iguales en una misma columna representan que no hay diferencia significativa entre los valores por la prueba de Duncan ( $\mathrm{p} \leq 0,05)$.

NOEC $=$ Concentración sin efecto observado. LOEC $=$ Concentración mínima con efecto observado. $\mathrm{F}=$ Prueba de Fisher. Sig. = Nivel de significancia.

Tabla 7. Toxicidad del extracto hexánico de Annona muricata, y Minthostachys mollis, sobre Chrysoperla externa (Neuroptera: Chrysopidae).

Table 7. Toxicity of the hexanic extract of Annona muricata, and Minthostachys mollis, against Chrysoperla externa (Neuroptera: Chrysopidae).

\begin{tabular}{lcccccc}
\hline & \multicolumn{5}{c}{ A. muricata } & \multicolumn{7}{c}{ M. mollis } \\
Concentración & $\mathbf{2 4} \mathbf{~ h}$ & $\mathbf{4 8} \mathbf{~ h}$ & $\mathbf{7 2} \mathbf{~ h}$ & $\mathbf{2 4} \mathbf{~ h}$ & $\mathbf{4 8} \mathbf{~ h}$ & $\mathbf{7 2} \mathbf{~ h}$ \\
\hline Control & $0 \mathrm{a}$ & $0 \mathrm{a}$ & $0 \mathrm{a}$ & $0 \mathrm{a}$ & $0 \mathrm{a}$ & $0 \mathrm{a}$ \\
$10 \%$ & $0 \mathrm{a}$ & $1,0 \mathrm{a}$ & $11,7 \mathrm{a}$ & $1,7 \mathrm{a}$ & $11,4 \mathrm{a}$ & $23,5 \mathrm{~b}$ \\
$20 \%$ & $0 \mathrm{a}$ & $6,2 \mathrm{a}$ & $17,6 \mathrm{a}$ & $1,7 \mathrm{a}$ & $8,8 \mathrm{a}$ & $26,4 \mathrm{~b}$ \\
NOEC (\%) & 20 & 20 & 20 & 20 & 20 & $<10$ \\
LOEC (\%) & $>20$ & $>20$ & $>20$ & $>20$ & $>20$ & 10 \\
F & 0,7 & 0,8 & 3,0 & 0,1 & 1,4 & 5,8 \\
Sig. & 0,5 & 0,4 & 0,1 & 0,8 & 0,2 & 0,02
\end{tabular}

Letras minúsculas iguales en una misma columna representan que no hay diferencia significativa entre los valores por la prueba de Duncan $(\mathrm{p} \leq 0,05)$.

NOEC $=$ Concentración $\sin$ efecto observado. LOEC $=$ Concentración mínima con efecto observado. $\mathrm{F}=$ Prueba de Fisher. Sig.= Nivel de significancia.

bilidad en una estrategia de manejo integrado de plagas (Sarmah et al. 2009, Iannacone et al. 2015, Liu et al. 2015)

\section{CONCLUSIONES}

El extracto acuoso de M. mollis y el extracto etanólico de C. quinoa, ambos al $20 \%$ de concentración podrían ser usados en un MIP de T. urticae, ya que fueron los únicos extractos botánicos que causaron mortalidad del ácaro en los bioensayos de toxicidad. Sin embargo, el extracto etanólico de $M$. mollis a las $72 \mathrm{~h}$ de exposición en
C. externa produjo una mortalidad muy elevada. El CRRS obtenido a las $48 \mathrm{~h}$ de exposición, indicó que el extracto acuoso de M. mollis al $20 \%$ de concentración podría ser empleado en un entorno natural donde coexistan o se liberen las larvas del primer instar de C. externa y las hembras adultas de T. urticae, debido a que este extracto no resultó ser riesgoso en la mortalidad del depredador, pero si tóxico en la plaga; sin embargo, aún es necesario realizar ensayos en campo para obtener información detallada acerca de la actividad acaricida de esta planta y su empleo en conjunto con otras estrategias de control dentro de un MIP. 
Tabla 8. Cociente relativo de riesgo de selectividad (CRRS) estimado de T. urticae/C. externa en mortalidades (\%) obtenidas con los extractos botánicos.

Table 8. Estimated relative risk quotient of selectivity (RRCS) of T. urticae / C. externa in mortalities $(\%)$ obtained with the botanical extracts.

\begin{tabular}{|c|c|c|c|c|}
\hline & \multicolumn{4}{|c|}{ Exposición $(48 \mathrm{~h})$} \\
\hline Extracto acuoso & & & & \\
\hline $10 \%$ & 1,4 & * & * & 15,6 \\
\hline $20 \%$ & 1,5 & 2,0 & 1,2 & 2,6 \\
\hline Riesgo al $10 \%$ & No & N.D & N.D & No \\
\hline Riesgo al 20 \% & No & No & No & No \\
\hline \multicolumn{5}{|l|}{ Extracto etanólico } \\
\hline $10 \%$ & * & $*$ & 2,5 & * \\
\hline $20 \%$ & * & 1,9 & 1,4 & 1,2 \\
\hline Riesgo al $10 \%$ & N.D & N.D & No & N.D \\
\hline Riesgo al $20 \%$ & N.D & No & No & No \\
\hline \multicolumn{5}{|l|}{ Extracto hexánico } \\
\hline $10 \%$ & * & $*$ & - & - \\
\hline $20 \%$ & * & 0,7 & - & - \\
\hline Riesgo al $10 \%$ & N.D & N.D & - & - \\
\hline Riesgo al $20 \%$ & N.D & $\mathrm{Si}$ & - & - \\
\hline Valor de comparación & 1 & 1 & 1 & 1 \\
\hline
\end{tabular}

Valores mayores a 1 del cociente de riesgo (CRRS) no provocaron riesgos en la mortalidad de C. externa.

${ }^{*}$ ) Valores que no afectaron la mortalidad de hembras adultas de T. urticae, pues sus resultados no son diferentes al control.

N.D $=$ No definido.

(-) Especies botánicas no tomadas en cuenta en la extracción con hexano.

Tabla 9. Dos criterios de elección para emplear los extractos botánicos en un posible Manejo Integrado de Plagas de T. urticae.

Table 9. Two selection criteria for using botanical extracts in a possible Integrated Pest Management of T. urticae.

\begin{tabular}{lccccc}
\hline Tratamiento & $\begin{array}{c}\text { Mortalidad en } \\
\text { ácaros (\%) }\end{array}$ & $\begin{array}{c}\text { Orden } \\
\mathbf{d e}>\mathbf{a}<\end{array}$ & $\begin{array}{c}\text { Cociente de } \\
\text { riesgo (CR) }\end{array}$ & $\begin{array}{c}\text { Orden } \\
\mathbf{d e}>\mathbf{a}<\end{array}$ & $\begin{array}{c}\Sigma \\
\text { (suma total) }\end{array}$ \\
\hline Extractos acuosos & & & & & \\
$20 \%$ A. muricata & 22,2 & 2,5 & 1,5 & 4 & 6,5 \\
$20 \%$ M. mollis & 29,6 & 1 & 2,0 & 2 & 3 \\
$20 \%$ L. mutabilis & 22,2 & 2,5 & 1,2 & 6 & 8,5 \\
$20 \%$ C. quinoa & 16,6 & 5 & 2,6 & 1 & 6 \\
Extractos etanólicos & $* *$ & $* *$ & $* *$ & $* *$ & $*$ \\
$20 \%$ A. muricata & 13,8 & 6,5 & 1,9 & 3 & 9,5 \\
$20 \%$ M. mollis & 13,8 & 6,5 & 1,4 & 5 & 11,5 \\
$20 \%$ L. mutabilis & 18,5 & 4 & 1,2 & 7 & 11 \\
$20 \%$ C. quinoa & $*$ & $*$ & $*$ & $*$ & $*$ \\
Extractos hexánicos & $* *$ & $* *$ & $*$ & $*$ \\
$20 \%$ A. muricata & $*$ & & & & \\
$20 \%$ M. mollis & $* *$ & & & & \\
\hline
\end{tabular}

$\left.{ }^{*}\right)$ Tomar los datos con precaución debido a que se observó $0 \%$ de mortalidad en T. urticae.

${ }^{* *}$ ) Valores que no afectaron la mortalidad de hembras adultas de T. urticae, pues sus resultados no son diferentes al control. 


\section{LITERATURA CITADA}

Aud, A.M., S. de Freitas, y L.R. Barbosa. 2001. Influencia de la dieta en la respuesta funcional de Chrysoperla externa (Hagen) (Neuroptera: Chrysopidae) alimentadas con Uroleucon ambrosiae (Thomas) (Hemiptera: Aphididae). Bol. San. Veg. Plagas 27:455-464.

Bahlai, C.A., Y. Xue, C. M. McCreary, A. W. Schaafsma, and R. H. Hallett. 2010. Choosing organic pesticides over synthetic pesticides may not effectively mitigate environmental risk in Soybeans. PLoS ONE 5:e11250.

Campos, E.V.R., J.L. de Oliveira, M. Pascoli, R. de Lima, and L.F. Fraceto. 2016. Neem oil and crop protection: from now to the future. Front. Plant Sci. 7:1494.

Carhuapoma Y.M., G.S. López, A.M. Roque, B. Velapatiño, C.C. Bell, y W.D. Whu. 2009. Actividad antibacteriana del aceite esencial de Minthostachys mollis Griseb "Ruyaq Muña". Ciencia e Investig. 12:83-89.

Castillo, G. 2004. Ensayos toxicológicos y métodos de evaluación de calidad de aguas. 189 p. Estandarización, intercalibración, resultados y aplicaciones. Centro Internacional de Investigaciones para el Desarrollo e Instituto Mexicano de Tecnología del Agua (IMTA), Jiutepec, México.

Coto-Alfaro, D., y J.L. Saunders. 2001. Insectos plaga de la guanábana (Anona muricata) en Costa Rica. Insect pests of soursop (Annona muricata) in Costa Rica. Manejo Integrado de Plagas 61:60-68.

Cronquist, A. 1981. An Integrated System of Classification of Flowering Plants. Columbia University Press, New York, USA.

Dadé, M., D.E. Fioravanti, G. Schinella, and H. Tournier. 2009. Total antioxidant capacity and polyphenol content of 21 aqueous extracts obtained from native plants of Traslasierra valley (Argentina). Bol. Latinoam. Caribe Plantas Med. Aromát.. 8:529-539.

de Moraes, G.J., y C.H.W. Flechtmann. 2008. Manual de Acarologia: Acarologia básica e ácaros de plantas cultivadas no Brasil. 308 p. Holos (ed.), Ribeirão Preto, São Paulo, Brasil.

Ferragut, F., y M. Santonja. 1989. Taxonomía y distribución de los ácaros del género Tetranychus Dufour 1832 (Acari: Tetranychidae), en España. Bol. San. Veg. Plagas 15:271-281.

Fusé, C.B., M.L. Villaverde, S.B. Padín, M. De Giusto, y Juaréz, M.P. 2013. Evaluación de la actividad insecticida de tierras de diatomeas de yacimientos argentinos. RIA. Rev. Investig. Agropecu. 39:207-213.
Gandarillas, H. 1967. Observaciones sobre la biología reproductiva de la quinua. Rev. Sayaña (Bolivia) 5:26-29.

Guzmán, B., R. Tenorio, D.L. Cruz, C. Espinal, J.A. Alvarado, and P. Mollinedo. 2015. Saponins from Chenopodium quinoa Willd and Chenopodium pallidicaule Aellen as biocontrollers of phytopathogen fungi and hemolysis agents. Rev. Bol. Quím. 32:8-14.

Hammond, G.B., I.D. Fernández, L.F. Villegas, and A.J. Vaisberg. 1998. A survey of traditional medicinal plants from the Callejón de Huaylas, Department of Ancash, Peru. J. Ethnopharmacol. 61:17-30.

Harder, M.J., V.E. Tello, and J.H. Giliomee. 2016. The acaricidal effect of ethanolic extracts of Chenopodium quinoa Willd. on Tetranychus urticae Koch (Acari: Tetranychidae). Afr. Entomol. 24:50-60.

Iannacone, J., y G. Lamas. 2002. Efecto de dos extractos botánicos y un insecticida convencional sobre el depredador Chrysoperla externa. Manejo Integrado de Plagas y Agroecología 65:92-101.

Iannacone, J., y G. Lamas. 2003a. Plantas biocidas usadas en el control de la polilla de la papa, Phthorimaea operculella (Zeller) (Lepidoptera: Gelechidae). Rev. Per. Ent. 43:79-87.

Iannacone, J., y G. Lamas. 2003b. Efectos toxicológicos de extractos de molle (Schinus molle) y lantana (Lantana camara) sobre Chrysoperla externa (Neuroptera: Chrysopidae), Trichogramma pintoi (Hymenoptera: Trichogrammatidae) y Copidosoma koehleri (Hymenoptera: Encyrtidae) en el Perú. Agric. Téc. 63:347-360.

Iannacone, J., y C. Quispe. 2004. Efecto insecticida de dos extractos vegetales sobre el gorgojo del maíz, Sitophilus zeamais Motschulsky, 1855 (Coleoptera: Curculionidae) en Perú. Rev. Per. Ent. 44:81-87.

Iannacone, J., and L. Alvariño. 2005. Selectividad del insecticida cartap empleando bioensayos con organismos no destinatarios. Ecol. Apl. 4:91-104.

Iannacone, J., L. Alvariño, J.C. Soto, y C. Salcedo. 2007. Efecto toxicológico del "Sachayoco", Paullinia clavigera (Sapindaceae) sobre Daphnia magna y sobre dos controladores biológicos de plagas agrícolas. J. Braz. Soc. Ecotoxicol. 2:15-25.

Iannacone, J., y L. Alvariño. 2010. Toxicidad de Schinus molle L. (Anacardiaceae) a cuatro controladores biológicos de plagas agrícolas en el Perú. Acta Zool. Mex. 26:603-615. 
Iannacone, J., L. Alvariño, M.I. La Torre, A. Guabloche, K. Ventura, J. Chero, et al. 2015. Toxicidad aguda y crónica de Tagetes elliptica (Asteraceae) y dimetoato sobre depredadores y parasitoides de plagas de importancia agrícola en Perú. Biologist (Lima) 13: 329-347.

Jacobsen, S.E., y A. Mujica. 2006. El tarwi (Lupinus mutabilis Sweet.) y sus parientes silvestres. p. 458-482. En Moraes, M., R., B. Øllgaard, L.P. Kvist, F. Borchsenius, y H. Balslev (eds.). Botánica Económica de los Andes Centrales. Universidad Mayor de San Andrés, La Paz, Bolivia.

Jeppson, L.R., H.H. Keifer, and E.W. Baker. 1975. Mites injurious to economic plants. 614 p. University California Press, Berkeley, California, USA.

Leatemia, J., and M. Isman. 2004. Toxicity and antifeedant activity of crude seed extracts of Annona squamosa (Annonaceae) against lepidopteran pests and natural enemies. Int. J. Trop. Insect Sci. 24:150-158.

Liu, Y.Q., B. Liu, A. Ali, S.P. Luo, Y.H. Lu, and G.M. Liang. 2015. Insecticide toxicity to Adelphocoris lineolatus (Hemiptera: Miridae) and its nymphal parasitoid Peristenus spretus (Hymenoptera: Braconidae). J. Econ. Entomol. 108:1779-1785.

Lock, O. 1994. Investigación Fitoquímica. Métodos en el estudio de productos naturales. 302 p. Editorial Pontificia Universidad Católica del Perú, Lima, Perú.

Maciel, A.G., J.S. Rodrigues, R.C. Trindade, E.S. Silva, A.E. Sant'Ana, and E.E. Lemos. 2015. Effect of Annona muricata L. (1753) (Annonaceae) seeds extracts on Tetranychus urticae (Koch, 1836) (Acari: Tetranychidae). Afr. J. Agric. Res. 10:4370-4375.

Mayo, L.D.V. 2013. Etnobotánica de plantas medicinales en el sector El Chispero en el municipio Piar, estado Monagas, año 2012. Trabajo de Grado como requisito para obtener el título de Ingeniero Agrónomo. Escuela de Ingeniería Agronómica, Universidad de Oriente Núcleo de Monagas, México.

Medina, P., F. Budia, P. Del Estal, and E. Viñuela. 2004. Influence of azadirachtin, a botanical insecticide, on Chrysoperla carnea (Stephens) reproduction: toxicity and ultrastructural approach. J. Econ. Entomol. 97:43-50.

Moura, A.P., G.A. Carvalho, and M. Botton. 2012. Residual effect of pesticides used in integrated apple production on Chrysoperla externa (Hagen) (Neuroptera: Chrysopidae) larvae. Chil. J. Agric. Res. 72:217.
Mujica, A., y S.E. Jacobsen. 2006. La quinua (Chenopodium quinoa Willd.) y sus parientes silvestres. p. 449-557. En Moraes, M., R., B. Øllgaard, L.P. Kvist, F. Borchsenius, y H. Balslev (eds.). Botánica Económica de los Andes Centrales. Universidad Mayor de San Andrés, La Paz, Bolivia.

Núñez, E. 1988. Ciclo biológico y crianza de Chrysoperla externa y Ceraeochrysa cincta (Neuroptera: Chrysopidae). Rev. Per. Ent. 31:76-82.

Parolin, P., C. Bresch, and C. Poncet. 2015. Biocontrol plants and functional diversity in biological control of the red spider mite Tetranychus urticae: A review. Int. J. Agr. Pol. Res. 3:198-212.

Russo, S., M. Grass, y S. Leicach. 2011. Efecto de extractos de Chenopodium album L. sobre los estados larval y adulto de Oryzaephilus surinamensis L. (Coleoptera: Silvanidae). Idesia 29:51-57.

Sarmah, M., A. Rahman, A.K. Phukan, and G. Gurusubramanian. 2009. Effect of aqueous plant extracts on tea red spider mite, Oligonychus coffeae, Nietner (Tetranychidae: Acarina) and Stethorus gilvifrons Mulsant. Afr. J. Biotechnol. 8:417-423.

Scandaliaris, M., E. Fuentes, and R.J. Lovey. 2007. Dos especies de Lamiáceas comercializadas en Córdoba (Argentina) bajo el nombre de "Peperina". Multequina 16:73-81.

Silva, R.A., G. Andrade, C. Freire, and D. Bastos. 2012. Effects of pesticides on eggs of Chrysoperla externa (Neuroptera: Chrysopidae) and consequences on subsequent development. Rev. Colomb. Entomol. 38:58-63.

Soto, J., y J. Iannacone. 2008. Efecto de dietas artificiales en la biología de adultos de Chrysoperla externa (Hagen, 1861) (Chrysopidae: Neuroptera). Acta Zool. Mex. 24:1-22.

Souza, A.P., M.R. Marqués, T.S. Mahmoud, B.A. Caputo, G.M. Canhete, C.B. Leite, et al. 2008. Bioprospecting insecticidal compounds from plants native to Mato Grosso do Sul, Brazil. Acta Bot. Bras. 22:1136-1140.

Stanley, J., and G. Preetha. 2016. Pesticide toxicity to arthropod predators: exposure, toxicity and risk assessment methodologies. p. 1-98. In J. Stanley, and G. Preetha (eds.) Pesticide toxicity to non-target organisms. Springer, Dordrecht.

Tava, A., and M. Odoardi. 1996. Saponins from Medicago spp.: chemical characterization and biological activity against insects. p. 97-109. In G.R. Waller, and T. Yamasaki (eds.) Saponins Used in Food and Agriculture. Ed. Plenum Press, New York, USA. 
Torrenegra-Alarcón, M., C. Granados-Conde, M. Durán-Lengua, G. León-Méndez, X. YáñezRueda, C. Martínez, et al. 2016. Composición química y actividad antibacteriana del aceite esencial de Minthostachys mollis. Orinoquia 20:69-74.

Van Leeuwen, T., J. Vontas, A. Tsagkarakou, W. Dermauw, and L. Tirry. 2010. Acaricide resistance mechanisms in the two-spotted spider mite Tetranychus urticae and other important Acari: a review. Insect Biochem Mol Biol. 40:563-572.

Woldemichael, G.M., and M. Wink. 2001. Identification and biological activities of triterpenoid saponins from Chenopodium quinoa. J. Agric. Food Chem. 49:2327-2332.

Zegarra, G. 2010. Actividad deterrente y acaricida de principios activos de quinuas amargas, aceites esenciales y tarwi. Tesis de Licenciatura en Química. Pontificia Universidad Católica del Perú, Lima, Perú. 